\title{
ALGEBRAIC RELATIONS AMONG SOME THETA FUNCTIONS ON THE BOUNDED SYMMETRIC DOMAIN OF TYPE $I_{r, r}$
}

\author{
Keiji MATSUMOTO
}

(Received 31 March 2005)

\begin{abstract}
We give algebraic relations among theta functions on the bounded symmetric domain of type $I_{r, r}$ : quadratic relations among those over the ring $\mathbb{Z}[i]$ of Gaussian integers, and over the ring $\mathbb{Z}[\sqrt{-2}]$, and cubic relations among those over the ring $\mathbb{Z}[\omega]$ of Eisenstein integers.
\end{abstract}

\section{Introduction}

Theta functions on the bounded symmetric domain $\mathbb{D}^{r}$ of type $I_{r, r}$ over the ring $\mathbb{Z}[i]$ of Gaussian integers and over the ring $\mathbb{Z}[\omega]$ of Eisenstein integers are utilized for constructions of modular forms on $\mathbb{D}^{r}$; refer to $[\mathbf{D K}, \mathbf{F}, \mathbf{F S}]$.

In this paper, we give quadratic relations among theta functions on $\mathbb{D}^{r}$ over $\mathbb{Z}[i]$ and over $\mathbb{Z}[\sqrt{-2}]$, and cubic relations among those over $\mathbb{Z}[\omega]$. Some of quadratic relations among theta functions over $\mathbb{Z}[i]$ were used when the author studied the period map for a four-parameter family of $K 3$-surfaces in [M], and the isomorphism between an octahedron in the 3-dimensional hyperbolic space to that in the 3-dimensional Euclid space in [MY], and the hyperbolic structure on the complement of the Whitehead link in [MNY]. Cubic relations among theta functions over $\mathbb{Z}[\omega]$ will help us to study periods of some families of algebraic varieties and the hyperbolic structure on the complement of the figure-eight knot.

2000 Mathematics Subject Classification: Primary 11F55.

Keywords and Phrases: theta functions.

This article is an invited contribution to a special issue of the Kyushu Journal of Mathematics commemorating the sixtieth volume. 


\section{Theta functions on the symmetric domain of type $I_{r, r}$}

The bounded symmetric domain $\mathbb{D}^{r}$ of type $I_{r, r}$ is defined as

$$
\mathbb{D}^{r}=\left\{\tau \in M_{r, r}(\mathbb{C}) \mid \frac{1}{2 i}\left(\tau-\tau^{*}\right) \text { is positive definite }\right\},
$$

where $i=\sqrt{-1}, X^{*}={ }^{\mathrm{t}} \bar{X}$ for a matrix $X$, and $M_{r, s}(R)$ is the set of $r \times s$ matrices over a ring $R$. Since

$$
\tau=\frac{1}{2}\left(\tau+\tau^{*}\right)+\frac{1}{2 i}\left(\tau-\tau^{*}\right) i
$$

any element of $\mathbb{D}^{r}$ can be expressed as the sum of a hermitian matrix and $i$ times a positive definite hermitian matrix. The unitary group

$$
U_{r, r}(\mathbb{C})=\left\{g \in G L_{2 r}(\mathbb{C}) \mid g J g^{*}=J=\left(\begin{array}{cc}
O & -I_{r} \\
I_{r} & O
\end{array}\right)\right\}
$$

and an involution $T$ act on $\mathbb{D}^{r}$ by

$$
g \cdot \tau=\left(g_{11} \tau+g_{12}\right)\left(g_{21} \tau+g_{22}\right)^{-1}, \quad T \cdot \tau={ }^{\mathrm{t}} \tau,
$$

where $\tau \in \mathbb{D}^{r}, g=\left(g_{i j}\right) \in U_{r, r}(\mathbb{C})$, and $g_{i j}$ are $r \times r$ matrices.

Let $d$ be a square-free positive integer and $\mathbb{Z}[\delta]$ be the ring of integers of the imaginary quadratic number field $\mathbb{Q}(\sqrt{-d})$, where

$$
\delta= \begin{cases}\sqrt{-d} & \text { if }-d \not \equiv 1 \bmod 4 \\ \frac{-1+\sqrt{-d}}{2} & \text { if }-d \equiv 1 \bmod 4\end{cases}
$$

The lattice $\mathbb{Z}[\delta]^{r}$ with the inner product

$$
\langle m, n\rangle= \begin{cases}\operatorname{Re}\left(m n^{*}\right) & \text { if }-d \not \equiv 1 \bmod 4, \\ 2 \operatorname{Re}\left(m n^{*}\right) & \text { if }-d \equiv 1 \bmod 4,\end{cases}
$$

is integral and its dual lattice $\left(\mathbb{Z}[\delta]^{r}\right)^{*}$ is $(1 / \sqrt{-d}) \mathbb{Z}[\delta]^{r}$.

Theta functions $\Theta_{\delta}\left(\begin{array}{l}a \\ b\end{array}\right)(\tau)$ on $\mathbb{D}^{r}$ over $\mathbb{Z}[\delta]$ are defined as

$$
\Theta_{\delta}\left(\begin{array}{l}
a \\
b
\end{array}\right)(\tau)= \begin{cases}\sum_{n \in \mathbb{Z}[\delta]^{r}} \mathbf{e}\left[(n+a) \tau(n+a)^{*}+2 \operatorname{Re}\left(n b^{*}\right)\right], & \text { if }-d \neq 1 \bmod 4, \\
\sum_{n \in \mathbb{Z}[\delta]^{r}} \mathbf{e}\left[2(n+a) \tau(n+a)^{*}+4 \operatorname{Re}\left(n b^{*}\right)\right], & \text { if }-d \equiv 1 \bmod 4,\end{cases}
$$

where $\tau \in \mathbb{D}^{r}, a, b \in \mathbb{Q}(\sqrt{-d})^{r}$ and $\mathbf{e}[x]=\exp [\pi i x]$. 
By definition, we have the following.

PROPOSITION 1. Theta functions $\Theta_{\delta}\left(\begin{array}{l}a \\ b\end{array}\right)(\tau)$ satisfy

(1) $\Theta_{\delta}\left(\begin{array}{c}\varepsilon a \\ \varepsilon b\end{array}\right)(\tau)=\Theta_{\delta}\left(\begin{array}{l}a \\ b\end{array}\right)(\tau)$ for a unit $\varepsilon \in \mathbb{Z}[\delta]$;

(2) $\Theta_{\delta}\left(\begin{array}{c}a+p \\ b+q\end{array}\right)(\tau)=\mathbf{e}[-2\langle p, b\rangle] \Theta_{\delta}\left(\begin{array}{l}a \\ b\end{array}\right)(\tau)$ for $p \in \mathbb{Z}[\delta]^{r}$ and $q \in(1 / \sqrt{-d}) \mathbb{Z}[\delta]^{r} \simeq$ $\left(\mathbb{Z}[\delta]^{r}\right)^{*}$;

(3) if $b \in(1 / 2 \sqrt{-d}) \mathbb{Z}[\delta]^{r}$, then $\Theta_{\delta}\left(\begin{array}{c}a \\ -b\end{array}\right)(\tau)=\Theta_{\delta}\left(\begin{array}{l}a \\ b\end{array}\right)(\tau)$;

(4) $\Theta_{\delta}\left(\begin{array}{l}a \\ b\end{array}\right)\left(g \tau g^{*}\right)=\Theta_{\delta}\left(\begin{array}{c}a g \\ b\left(g^{*}\right)^{-1}\end{array}\right)(\tau)$ for any $g \in G L_{r}(\mathbb{Z}[\delta])$;

(5) $\Theta_{\delta}\left(\begin{array}{l}a \\ b\end{array}\right)(T \cdot \tau)=\Theta_{\delta}\left(\begin{array}{l}\bar{a} \\ \bar{b}\end{array}\right)(\tau)$.

\section{Quadratic relations among theta functions over $\mathbb{Z}[i]$}

In this section, we give quadratic relations among theta functions over $\mathbb{Z}[i]$ :

$$
\Theta\left(\begin{array}{l}
a \\
b
\end{array}\right)(\tau)=\Theta_{i}\left(\begin{array}{l}
a \\
b
\end{array}\right)(\tau)=\sum_{n \in \mathbb{Z}[i]^{r}} \mathbf{e}\left[(n+a) \tau(n+a)^{*}+2 \operatorname{Re}\left(n b^{*}\right)\right] .
$$

THEOREM 1. We have

$$
\begin{aligned}
& 2^{r} \Theta\left(\begin{array}{l}
a_{1}+a_{2} \\
b_{1}+b_{2}
\end{array}\right)(\tau) \Theta\left(\begin{array}{l}
a_{1}-a_{2} \\
b_{1}-b_{2}
\end{array}\right)(\tau) \\
= & \sum_{e, f \in R_{1+i}^{r}} \mathbf{e}\left[2 \operatorname{Re}\left((1+i)\left(b_{1}+b_{2}\right) e^{*}\right)\right] \Theta\left(\begin{array}{l}
e+(1+i) a_{1} \\
f+(1+i) b_{1}
\end{array}\right)(\tau) \Theta\left(\begin{array}{l}
e+(1+i) a_{2} \\
f+(1+i) b_{2}
\end{array}\right)(\tau),
\end{aligned}
$$

where $R_{1+i}=\frac{\mathbb{Z}[i]}{1+i} / \mathbb{Z}[i]$. In particular,

$$
2^{r} \Theta\left(\begin{array}{l}
a \\
b
\end{array}\right)(\tau)^{2}=\sum_{e, f \in R_{1+i}^{r}} \mathbf{e}\left[2 \operatorname{Re}\left((1+i) b e^{*}\right)\right] \Theta\left(\begin{array}{c}
e+(1+i) a \\
f+(1+i) b
\end{array}\right)(\tau) \Theta\left(\begin{array}{l}
e \\
f
\end{array}\right)(\tau) .
$$

Proof. For the lattice $L_{1}=M_{2, r}(\mathbb{Z}[i])$, we set $L_{2}=U L_{1}$ and $L=L_{1}+L_{2}$, where

$$
U=\frac{1+i}{2}\left(\begin{array}{cc}
1 & 1 \\
1 & -1
\end{array}\right)
$$

note that $U U^{*}=I_{2}$ and $U^{2}=i I_{2}$. Since

$$
L_{2}=\left\{M=\left(\begin{array}{l}
m_{1} \\
m_{2}
\end{array}\right) \in \frac{1+i}{2} M_{2, r}(\mathbb{Z}[i]) \mid m_{1}+m_{2} \in(1+i) \mathbb{Z}[i]^{r}\right\},
$$




$$
L=\left\{M=\left(\begin{array}{l}
m_{1} \\
m_{2}
\end{array}\right) \in \frac{1+i}{2} M_{2, r}(\mathbb{Z}[i]) \mid m_{1}+m_{2} \in \mathbb{Z}[i]^{r}\right\},
$$

we have $\left[L: L_{1}\right]=\left[L: L_{2}\right]=2^{r}$,

$$
\begin{gathered}
L / L_{2} \simeq L_{1} /\left(L_{1} \cap L_{2}\right)=\left\{P=\left(\begin{array}{l}
p \\
0
\end{array}\right) \mid p \in\{0,1\}^{r}\right\}, \\
L / L_{1} \simeq L_{2} /\left(L_{1} \cap L_{2}\right)=\left\{U P=\frac{1+i}{2}\left(\begin{array}{l}
p \\
p
\end{array}\right) \mid p \in\{0,1\}^{r}\right\} .
\end{gathered}
$$

For any $Q \in L_{1}$, the map $\chi_{Q}: L / L_{1} \ni M \mapsto \mathbf{e}\left[2 \operatorname{Re}\left(\operatorname{tr}\left(M Q^{*}\right)\right)\right] \in \mathbb{C}^{\times}$is an element of the group $\left(L / L_{1}\right)^{\times}$of homomorphisms from $L / L_{1}$ to $\mathbb{C}^{\times}$, where $\operatorname{tr}(X)$ denotes the trace of a square matrix $X$. It is easy to see that $\chi_{Q}=1$ if and only if $Q \in L_{1} \cap L_{2}$. These imply that

$$
\left(L / L_{1}\right)^{\times}=\left\{\chi_{Q} \mid Q \in L_{1} /\left(L_{1} \cap L_{2}\right)\right\} .
$$

Apply Lemma 1 of [I, Ch. IV] for $L, L_{1}, L_{2}$, and for the function

$$
\psi(X)=\mathbf{e}\left[\operatorname{tr}\left\{(X+A) \tau(X+A)^{*}+2 \operatorname{Re}\left(X B^{*}\right)\right\}\right]
$$

where $\tau \in \mathbb{D}^{r}$,

$$
A=\left(\begin{array}{l}
a_{1}+a_{2} \\
a_{1}-a_{2}
\end{array}\right), \quad B=\left(\begin{array}{l}
b_{1}+b_{2} \\
b_{1}-b_{2}
\end{array}\right) .
$$

Then we have

$$
\left[L: L_{1}\right] \sum_{X \in L_{1}} \psi(X)=\sum_{P, Q \in L / L_{2}}\left(\sum_{M \in L_{2}} \chi_{Q}(M+P) \psi(M+P)\right) .
$$

The left-hand side of (1) is equal to $2^{r} \Theta\left(\begin{array}{l}a_{1}+a_{2} \\ b_{1}+b_{2}\end{array}\right)(\tau) \Theta\left(\begin{array}{l}a_{1}-a_{2} \\ b_{1}-b_{2}\end{array}\right)(\tau)$. The right-hand side of (1) is equal to

$$
\begin{array}{rl}
\sum_{P, Q \in L / L_{2}} \sum_{M \in L_{2}} & \mathbf{e}\left[2 \operatorname{Re}\left\{\operatorname{tr}\left((M+P) Q^{*}\right)\right\}\right] \\
\times \mathbf{e}\left[\operatorname{tr}\left\{(M+P+A) \tau(M+P+A)^{*}+2 \operatorname{Re}\left((M+P) B^{*}\right)\right\}\right]
\end{array}
$$




$$
\begin{aligned}
& =\sum_{P, Q \in L / L_{2}} \sum_{N \in L_{1}} \begin{array}{l}
\mathbf{e}\left[2 \operatorname{Re}\left\{\operatorname{tr}\left(U^{*} N Q^{*}\right)\right\}\right] \mathbf{e}\left[2 \operatorname{Re}\left\{\operatorname{tr}\left(P Q^{*}\right)\right\}\right] \mathbf{e}\left[2 \operatorname{Re}\left\{\operatorname{tr}\left(P B^{*}\right)\right\}\right] \\
\times \mathbf{e}\left[\operatorname{tr}\left\{\left(U^{*} N+P+A\right) \tau\left(U^{*} N+P+A\right)^{*}+2 \operatorname{Re}\left(U^{*} N B^{*}\right)\right\}\right] \\
=\sum_{P, Q \in L / L_{2}} \sum_{N \in L_{1}} \mathbf{e}\left[2 \operatorname{Re}\left\{\operatorname{tr}\left(P B^{*}\right)\right\}\right] \\
\times \mathbf{e}\left[\operatorname{tr}\left\{(N+U P+U A) \tau(N+U P+U A)^{*}+2 \operatorname{Re}\left(N(U B+U Q)^{*}\right)\right\}\right]
\end{array}
\end{aligned}
$$

since $\operatorname{tr}\left(U X U^{*}\right)=\operatorname{tr}(X)$ for any $(2 \times 2)$-matrix $X$, and $\operatorname{tr}\left(P Q^{*}\right) \in \mathbb{Z}[i]$. Put

$$
U P=\left(\begin{array}{l}
e \\
e
\end{array}\right) \quad \text { and } \quad U Q=\left(\begin{array}{l}
f \\
f
\end{array}\right), \quad \text { where } e, f \in \frac{1+i}{2} \mathbb{Z}[i]^{r} .
$$

Note that

$$
U A=(1+i)\left(\begin{array}{l}
a_{1} \\
a_{2}
\end{array}\right), \quad U B=(1+i)\left(\begin{array}{l}
b_{1} \\
b_{2}
\end{array}\right)
$$

and that

$$
\operatorname{Re}\left(\operatorname{tr}\left(P B^{*}\right)\right)=\operatorname{Re}\left(\operatorname{tr}\left(B P^{*}\right)\right)=\operatorname{Re}\left((1+i)\left(b_{1}+b_{2}\right) e^{*}\right) .
$$

Thus, the right-hand side of (1) is equal to

$$
\sum_{e, f \in R_{1+i}^{r}} \mathbf{e}\left[2 \operatorname{Re}\left((1+i)\left(b_{1}+b_{2}\right) e^{*}\right)\right] \Theta\left(\begin{array}{c}
e+(1+i) a_{1} \\
f+(1+i) b_{1}
\end{array}\right)(\tau) \Theta\left(\begin{array}{c}
e+(1+i) a_{2} \\
f+(1+i) b_{2}
\end{array}\right)(\tau) .
$$

By putting $a_{1}=a, b_{1}=b$ and $a_{2}=b_{2}=0$, we get the second equality of the theorem.

Remark 1. The lattices $L$ and $L_{1} \cap L_{2}$ are isomorphic to $\left(D_{4}^{*}\right)^{r}$ and $\left(D_{4}\right)^{r}$, respectively, where $D_{4}$ is the root lattice of type $D_{4}$, and $D_{4}^{*}$ is its dual.

Remark 2. Refer to [M, MNY, MY] for applications of this theorem.

\section{Quadratic relations among theta functions over $\mathbb{Z}[\sqrt{-2}]$}

In this section, we give quadratic relations among theta functions over $\mathbb{Z}[\sqrt{-2}]$ :

$$
\Theta\left(\begin{array}{l}
a \\
b
\end{array}\right)(\tau)=\Theta_{\sqrt{-2}}\left(\begin{array}{l}
a \\
b
\end{array}\right)(\tau)=\sum_{n \in \mathbb{Z}[\sqrt{-2}]^{r}} \mathbf{e}\left[(n+a) \tau(n+a)^{*}+2 \operatorname{Re}\left(n b^{*}\right)\right] .
$$


THEOREM 2. We have

$$
\begin{aligned}
& 2^{r} \Theta\left(\begin{array}{l}
a_{1}+a_{2} \\
b_{1}+b_{2}
\end{array}\right)(\tau) \Theta\left(\begin{array}{l}
a_{1}-a_{2} \\
b_{1}-b_{2}
\end{array}\right)(\tau) \\
= & \sum_{e, f \in R_{\sqrt{-2}}^{r}} \mathbf{e}\left[-2 \operatorname{Re}\left(\sqrt{-2} e\left(b_{1}+b_{2}\right)^{*}\right)\right] \prod_{j=1}^{2} \Theta\left(\begin{array}{c}
e+\sqrt{-2} a_{j} \\
\frac{f}{\sqrt{-2}}+\sqrt{-2} b_{j}
\end{array}\right)(\tau),
\end{aligned}
$$

where $R_{\sqrt{-2}}=\frac{\mathbb{Z}[\sqrt{-2}]}{\sqrt{-2}} / \mathbb{Z}[-2]$. In particular,

$$
2^{r} \Theta\left(\begin{array}{l}
a \\
b
\end{array}\right)(\tau)^{2}=\sum_{e, f \in R_{\sqrt{-2}}^{r}} \mathbf{e}\left[-2 \operatorname{Re}\left(\sqrt{-2} e b^{*}\right)\right] \Theta\left(\begin{array}{c}
e+\sqrt{-2} a \\
\frac{f}{\sqrt{-2}}+\sqrt{-2} b
\end{array}\right)(\tau) \Theta\left(\begin{array}{c}
e \\
f \\
\frac{\sqrt{-2}}{2}
\end{array}\right)(\tau) .
$$

Proof. Set

$$
\begin{aligned}
L_{1} & =M_{2, r}(\mathbb{Z}[\sqrt{-2}]), \quad U=\frac{1}{\sqrt{-2}}\left(\begin{array}{cc}
1 & 1 \\
1 & -1
\end{array}\right), \\
L_{2} & =U L_{1}=\left\{M=\left(\begin{array}{l}
m_{1} \\
m_{2}
\end{array}\right) \in \frac{1}{\sqrt{-2}} L_{1} \mid m_{1}+m_{2} \in \sqrt{-2} \mathbb{Z}[\sqrt{-2}]^{r}\right\}, \\
L & =L_{1}+L_{2}=\left\{M=\left(\begin{array}{l}
m_{1} \\
m_{2}
\end{array}\right) \in \frac{1}{\sqrt{-2}} L_{1} \mid m_{1}+m_{2} \in \mathbb{Z}[\sqrt{-2}]^{r}\right\}, \\
L / L_{2} & \simeq L_{1} /\left(L_{1} \cap L_{2}\right)=\left\{P=\left(\begin{array}{l}
p \\
0
\end{array}\right) \mid p \in\{0,1\}^{r}\right\}, \\
L / L_{1} & \simeq L_{2} /\left(L_{1} \cap L_{2}\right)=\left\{U P=\frac{1}{\sqrt{-2}}\left(\begin{array}{l}
p \\
p
\end{array}\right) \mid p \in\{0,1\}^{r}\right\}, \\
\left(L / L_{1}\right)^{\times} & =\left\{\begin{array}{l}
\chi Q \\
\chi
\end{array}\left|L / L_{1} \ni M \mapsto \mathbf{e}\left[2 \operatorname{Re}\left(\frac{\operatorname{tr}\left(M Q^{*}\right)}{\sqrt{-2}}\right)\right] \in \mathbb{C}^{\times}\right| Q \in L_{1} /\left(L_{1} \cap L_{2}\right)\right\}, \\
\psi(X) & =\mathbf{e}\left[\operatorname{tr}\left\{(X+A) \tau(X+A)^{*}+2 \operatorname{Re}\left(X B^{*}\right)\right\}\right], \\
A & =\left(\begin{array}{l}
a_{1}+a_{2} \\
a_{1}-a_{2}
\end{array}\right), \quad B=\left(\begin{array}{l}
b_{1}+b_{2} \\
b_{1}-b_{2}
\end{array}\right),
\end{aligned}
$$

and follow the proof of Theorem 1. 
To simplify quadratic relations among $\Theta_{\sqrt{-2}}\left(\begin{array}{l}a \\ b\end{array}\right)(\tau)$, we put

$$
\begin{aligned}
\Theta_{\sqrt{-2}}\left[\begin{array}{l}
a \\
b
\end{array}\right](\tau) & =\Theta_{\sqrt{-2}}\left(\begin{array}{c}
\frac{a}{\sqrt{-2}} \\
\frac{b}{2}
\end{array}\right)(\tau) \\
& =\sum_{n \in \mathbb{Z}[\sqrt{-2}]^{r}} \mathbf{e}\left[\left(n+\frac{a}{\sqrt{-2}}\right) \tau\left(n+\frac{a}{\sqrt{-2}}\right)^{*}+\operatorname{Re}\left(n b^{*}\right)\right] .
\end{aligned}
$$

They satisfy:

(1) $\Theta_{\sqrt{-2}}\left[\begin{array}{c}-a \\ -b\end{array}\right](\tau)=\Theta_{\sqrt{-2}}\left[\begin{array}{l}a \\ b\end{array}\right](\tau)$;

(2) $\Theta_{\sqrt{-2}}\left[\begin{array}{l}a+\sqrt{-2} p \\ b+\sqrt{-2} q\end{array}\right](\tau)=\mathbf{e}\left[-\operatorname{Re}\left(p b^{*}\right)\right] \Theta_{\sqrt{-2}}\left[\begin{array}{l}a \\ b\end{array}\right](\tau) \quad$ for $p, q \in \mathbb{Z}[\sqrt{-2}]^{r}$;

(3) if $b \in \frac{\mathbb{Z}[\sqrt{-2}]^{r}}{\sqrt{-2}}$, then $\Theta_{\sqrt{-2}}\left[\begin{array}{c}a \\ -b\end{array}\right](\tau)=\Theta_{\sqrt{-2}}\left[\begin{array}{l}a \\ b\end{array}\right](\tau)$;

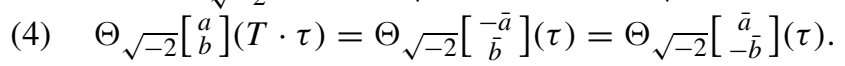

COROllary 1. For $a, b \in \mathbb{Z}[\sqrt{-2}]^{r}$, we have

$$
2^{r} \Theta_{\sqrt{-2}}^{2}\left[\begin{array}{l}
a \\
b
\end{array}\right](\tau)=\sum_{p, q \in\{0,1\}^{r}}(-1)^{\operatorname{Re}\left(p b^{*}+a q^{*}\right)} \Theta_{\sqrt{-2}}^{2}\left[\begin{array}{l}
p \\
q
\end{array}\right](\tau) .
$$

Proof. Theorem 2 implies that

$$
\begin{aligned}
& 2^{r} \Theta_{\sqrt{-2}}^{2}\left[\begin{array}{l}
a \\
b
\end{array}\right](\tau) 2^{r} \Theta\left(\begin{array}{c}
\frac{a}{\sqrt{-2}} \\
\frac{b}{2}
\end{array}\right)(\tau)^{2} \\
& =\sum_{e, f \in R_{\sqrt{-2}}^{r}} \mathbf{e}\left[-\operatorname{Re}\left(\sqrt{-2} e b^{*}\right)\right] \Theta\left(\begin{array}{c}
e+a \\
\frac{f}{\sqrt{-2}}-\frac{b}{\sqrt{-2}}
\end{array}\right)(\tau) \Theta\left(\begin{array}{c}
e \\
\frac{f}{\sqrt{-2}}
\end{array}\right)(\tau) \\
& =\sum_{e, f \in R_{\sqrt{-2}}^{r}} \mathbf{e}\left[-\operatorname{Re}\left(\sqrt{-2} e b^{*}\right)\right] \Theta_{\sqrt{-2}}\left[\begin{array}{c}
\sqrt{-2}(e+a) \\
-\sqrt{-2}(f-b)
\end{array}\right](\tau) \Theta_{\sqrt{-2}}\left[\begin{array}{c}
\sqrt{-2} e \\
-\sqrt{-2} f
\end{array}\right](\tau) \\
& =\sum_{p, q \in\{0, \pm 1\}^{r}} \mathbf{e}\left[-\operatorname{Re}\left(p b^{*}+a q^{*}\right)\right] \Theta_{\sqrt{-2}}^{2}\left[\begin{array}{l}
p \\
q
\end{array}\right](\tau),
\end{aligned}
$$

where we put $p=\sqrt{-2} e$ and $q=-\sqrt{-2} f$.

The domain $\mathbb{D}^{r}$ is nothing but the upper half plane $\mathbb{H}$ for $r=1$. There are four $\Theta_{\sqrt{-2}}\left[\begin{array}{l}a \\ b\end{array}\right]$ on $\mathbb{H}$ over $\mathbb{Z}[\sqrt{-2}]$ for $a, b \in\{0,1\}$ :

$$
\Theta_{\sqrt{-2}}\left[\begin{array}{l}
0 \\
0
\end{array}\right], \quad \Theta_{\sqrt{-2}}\left[\begin{array}{l}
1 \\
0
\end{array}\right], \quad \Theta_{\sqrt{-2}}\left[\begin{array}{l}
0 \\
1
\end{array}\right], \quad \Theta_{\sqrt{-2}}\left[\begin{array}{l}
1 \\
1
\end{array}\right] .
$$


Corollary 1 implies the quadratic relation among them:

$$
\Theta_{\sqrt{-2}}^{2}\left[\begin{array}{l}
1 \\
1
\end{array}\right](\tau)=\Theta_{\sqrt{-2}}^{2}\left[\begin{array}{l}
0 \\
0
\end{array}\right](\tau)-\Theta_{\sqrt{-2}}^{2}\left[\begin{array}{l}
1 \\
0
\end{array}\right](\tau)-\Theta_{\sqrt{-2}}^{2}\left[\begin{array}{l}
0 \\
1
\end{array}\right](\tau) .
$$

For $r=2$, there are 16 non-zero $\Theta_{\sqrt{-2}}\left[\begin{array}{l}a \\ b\end{array}\right]$ on $\mathbb{D}^{2}$ over $\mathbb{Z}[\sqrt{-2}]$ for $a, b \in\{0,1\}^{2}$. Corollary 1 implies linear relations among their squares. The ten $\Theta_{\sqrt{-2}}^{2}\left[\begin{array}{l}a \\ b\end{array}\right]$ satisfying $\operatorname{Re}\left(a b^{*}\right) \in 2 \mathbb{Z}$ are linearly independent in these relations. The six $\Theta_{\sqrt{-2}}^{2}\left[\begin{array}{l}a \\ b\end{array}\right]$ satisfying $\operatorname{Re}\left(a b^{*}\right) \notin 2 \mathbb{Z}$ are expressed as

$$
\Theta_{\sqrt{-2}}^{2}\left[\begin{array}{l}
a \\
b
\end{array}\right]=\frac{1}{2} \sum_{p, q \in\{0,1\}^{2}}^{p q^{*} \in 2 \mathbb{Z}}(-1)^{p b^{*}+a q^{*}} \Theta_{\sqrt{-2}}^{2}\left[\begin{array}{l}
p \\
q
\end{array}\right] .
$$

For $r=3$, there are 64 non-zero $\Theta_{\sqrt{-2}}\left[\begin{array}{l}a \\ b\end{array}\right]$ on $\mathbb{D}^{3}$ over $\mathbb{Z}[\sqrt{-2}]$ for $a, b \in\{0,1\}^{3}$. Corollary 1 implies linear relations among their squares. The $36 \Theta_{\sqrt{-2}}^{2}\left[\begin{array}{l}a \\ b\end{array}\right]$ satisfying $\operatorname{Re}\left(a b^{*}\right) \in 2 \mathbb{Z}$ are linearly independent in these relations. The $28 \Theta_{\sqrt{-2}}^{2}\left[\begin{array}{l}a \\ b\end{array}\right]$ satisfying $\operatorname{Re}\left(a b^{*}\right) \notin 2 \mathbb{Z}$ are expressed as

$$
\Theta_{\sqrt{-2}}^{2}\left[\begin{array}{l}
a \\
b
\end{array}\right]=\frac{1}{2^{2}} \sum_{p, q \in\{0,1\}^{3}}^{p q^{*} \in 2 \mathbb{Z}}(-1)^{p b^{*}+a q^{*}} \Theta_{\sqrt{-2}}^{2}\left[\begin{array}{l}
p \\
q
\end{array}\right] .
$$

\section{Cubic relations among theta functions over $\mathbb{Z}[\omega]$}

In this section, we give cubic relations among theta functions over $\mathbb{Z}[\omega]$ :

$$
\Theta\left(\begin{array}{l}
a \\
b
\end{array}\right)(\tau)=\Theta_{\omega}\left(\begin{array}{l}
a \\
b
\end{array}\right)(\tau)=\sum_{n \in \mathbb{Z}[\omega]^{r}} \mathbf{e}\left[2(n+a) \tau(n+a)^{*}+4 \operatorname{Re}\left(n b^{*}\right)\right] .
$$

THEOREM 3. We have

$$
\begin{aligned}
& 3^{r} \Theta\left(\begin{array}{l}
a_{1}+a_{2}+a_{3} \\
b_{1}+b_{2}+b_{3}
\end{array}\right)(\tau) \Theta\left(\begin{array}{l}
a_{1}+\omega a_{2}+\omega^{2} a_{3} \\
b_{1}+\omega b_{2}+\omega^{2} b_{3}
\end{array}\right)(\tau) \Theta\left(\begin{array}{l}
a_{1}+\omega^{2} a_{2}+\omega a_{3} \\
b_{1}+\omega^{2} b_{2}+\omega b_{3}
\end{array}\right)(\tau) \\
= & \sum_{e, f \in R_{\sqrt{-3}}^{r}} \mathbf{e}\left[-4 \operatorname{Re}\left(\sqrt{-3} e\left(b_{1}+b_{2}+b_{3}\right)^{*}\right)\right] \prod_{j=1}^{3} \Theta\left(\begin{array}{c}
e+\sqrt{-3} a_{j} \\
\frac{f}{\sqrt{-3}}+\sqrt{-3} b_{j}
\end{array}\right)(\tau),
\end{aligned}
$$


where $R_{\sqrt{-3}}=\frac{\mathbb{Z}[\omega]}{\sqrt{-3}} / \mathbb{Z}[\omega]$. In particular,

$$
\begin{aligned}
& 3^{r} \Theta\left(\begin{array}{l}
a \\
b
\end{array}\right)(\tau)^{3} \\
= & \sum_{e, f \in R_{\sqrt{-3}}^{r}} \mathbf{e}\left[-4 \operatorname{Re}\left(\sqrt{-3} e b^{*}\right)\right] \Theta\left(\begin{array}{l}
e+\sqrt{-3} a \\
\frac{f}{\sqrt{-3}}+\sqrt{-3} b
\end{array}\right)(\tau) \Theta\left(\begin{array}{c}
e \\
\frac{f}{\sqrt{-3}}
\end{array}\right)(\tau)^{2} .
\end{aligned}
$$

Proof. Let $L_{1}$ be $M_{3, r}(\mathbb{Z}[\omega])$ and $L_{2}$ be $V L_{1}$, where

$$
V=\frac{1}{\sqrt{-3}}\left(\begin{array}{ccc}
1 & 1 & 1 \\
1 & \omega & \omega^{2} \\
1 & \omega^{2} & \omega
\end{array}\right)
$$

note that $V V^{*}=I_{3}, V^{2}=-\left(\begin{array}{cc}1 & 1 \\ & 1\end{array}\right) \in G L_{3}(\mathbb{Z}[\omega])$. Note also that

$$
\begin{gathered}
L_{2}=\left\{M=\left(\begin{array}{l}
m_{1} \\
m_{2} \\
m_{3}
\end{array}\right) \in \frac{1}{\sqrt{-3}} M_{3, r}(\mathbb{Z}[\omega]) \mid \begin{array}{c}
m_{j}-m_{k} \in \mathbb{Z}[\omega]^{r}(1 \leq j, k \leq 3) \\
m_{1}+m_{2}+m_{3} \in \sqrt{-3} \mathbb{Z}[\omega]^{r}
\end{array}\right\}, \\
L_{1} \cap L_{2}=\left\{N=\left\{\begin{array}{l}
n_{1} \\
n_{2} \\
n_{3}
\end{array}\right) \in M_{3, r}(\mathbb{Z}[\omega]) \mid n_{1}+n_{2}+n_{3} \in \sqrt{-3} \mathbb{Z}[\omega]^{r}\right\},
\end{gathered}
$$

and that the lattice $L$ generated by $L_{1}$ and $L_{2}$ is

$$
L=\left\{M=\left(\begin{array}{l}
m_{1} \\
m_{2} \\
m_{3}
\end{array}\right) \in \frac{1}{\sqrt{-3}} M_{3, r}(\mathbb{Z}[\omega]) \mid m_{j}-m_{k} \in \mathbb{Z}[\omega]^{r}(1 \leq j, k \leq 3)\right\} .
$$

Thus, we have

$$
\left[L: L_{1}\right]=\left[L: L_{2}\right]=\left[L_{1}: L_{1} \cap L_{2}\right]=\left[L_{2}: L_{1} \cap L_{2}\right]=3^{r},
$$

and so the representatives of $L / L_{2} \simeq L_{1} /\left(L_{1} \cap L_{2}\right)$ and those of $L / L_{1} \simeq L_{2} /\left(L_{1} \cap L_{2}\right)$ are given by

$P=\left(\begin{array}{l}p \\ 0 \\ 0\end{array}\right) \in M_{3, r}(\mathbb{Z}[\omega]), \quad V P=\frac{1}{\sqrt{-3}}\left(\begin{array}{l}p \\ p \\ p\end{array}\right) \in \frac{1}{\sqrt{-3}} M_{3, r}(\mathbb{Z}[\omega]), \quad p \in\{0, \pm 1\}^{r}$,

respectively. 
For any $Q \in L_{1}$, the map $\chi_{Q}: L / L_{1} \ni N \mapsto \mathbf{e}\left[4 \operatorname{Re}\left((1 / \sqrt{-3}) \operatorname{tr}\left(N Q^{*}\right)\right)\right] \in \mathbb{C}^{\times}$ is an element of the group $\left(L / L_{1}\right)^{\times}$of homomorphisms from $L / L_{1}$ to $\mathbb{C}^{\times}$. Since any element $L$ can be expressed as $N_{1}+V N_{2}$ for $N_{1}, N_{2} \in L_{1}$, and an element $Q$ of $L_{1}$ belongs to $L_{1} \cap L_{2}$ if and only if $V^{*} Q$ belongs to $L_{1}$, we have

$$
\begin{aligned}
\mathbf{e}\left[4 \operatorname{Re}\left(\frac{1}{\sqrt{-3}} \operatorname{tr}\left(\left(N_{1}+V N_{2}\right) Q^{*}\right)\right)\right] & =\mathbf{e}\left[4 \operatorname{Re}\left(\frac{1}{\sqrt{-3}} \operatorname{tr}\left(V N_{2} Q^{*}\right)\right)\right] \\
= & \mathbf{e}\left[4 \operatorname{Re}\left(\frac{1}{\sqrt{-3}} \operatorname{tr}\left(N_{2} Q^{*} V\right)\right)\right]=\mathbf{e}\left[4 \operatorname{Re}\left(\frac{1}{\sqrt{-3}} \operatorname{tr}\left(N_{2}\left(V^{*} Q\right)^{*}\right)\right)\right]=1
\end{aligned}
$$

for any $Q \in L_{1} \cap L_{2}$. Thus, $\chi_{Q}=1$ if and only if $Q \in L_{1} \cap L_{2}$; this implies that

$$
\left(L / L_{1}\right)^{\times}=\left\{\chi_{Q} \mid Q \in L_{1} /\left(L_{1} \cap L_{2}\right)\right\} .
$$

We follow the proof of Theorem 1 for $L, L_{1}, L_{2}$ and for the function

$$
\psi(X)=\mathbf{e}\left[\operatorname{tr}\left\{(X+A) \tau(X+A)^{*}+4 \operatorname{Re}\left(X B^{*}\right)\right\}\right]
$$

where

$$
\begin{gathered}
\tau \in \mathbb{D}^{r}, \quad A=\left(\begin{array}{c}
a_{1}+a_{2}+a_{3} \\
a_{1}+\omega^{2} a_{2}+\omega a_{3} \\
a_{1}+\omega a_{2}+\omega^{2} a_{3}
\end{array}\right), \\
B=\left(\begin{array}{c}
b_{1}+b_{2}+b_{3} \\
b_{1}+\omega^{2} b_{2}+\omega b_{3} \\
b_{1}+\omega b_{2}+\omega^{2} b_{3}
\end{array}\right), \quad a_{j}, b_{j} \in \mathbb{Q}(\omega)^{r} .
\end{gathered}
$$

Then we have

$$
\left[L: L_{1}\right] \sum_{N \in L_{1}} \psi(N)=\sum_{P, Q \in L / L_{2}} \sum_{M \in L_{2}} \chi_{Q}(M+P) \psi(M+P) .
$$

It is easy to see that the left-hand side of (2) becomes

$$
3^{r} \Theta\left(\begin{array}{l}
a_{1}+a_{2}+a_{3} \\
b_{1}+b_{2}+b_{3}
\end{array}\right)(\tau) \Theta\left(\begin{array}{l}
a_{1}+\omega a_{2}+\omega^{2} a_{3} \\
b_{1}+\omega b_{2}+\omega^{2} b_{3}
\end{array}\right)(\tau) \Theta\left(\begin{array}{l}
a_{1}+\omega^{2} a_{2}+\omega a_{3} \\
b_{1}+\omega^{2} b_{2}+\omega b_{3}
\end{array}\right)(\tau) .
$$

For any $M \in L_{2}$, there exists $N^{\prime} \in L_{1}$ such that $M=V N^{\prime}$. Since $M=V N^{\prime}=$ $V^{*}\left(V^{2} N^{\prime}\right)$ and $V^{2} \in G L_{3}(\mathbb{Z}[\omega])$, there also exists $N \in L_{1}$ such that $M=V^{*} N$. 
The right-hand side of (2) is

$$
\begin{aligned}
& \sum_{P, Q \in L / L_{2}} \sum_{M \in L_{2}} \mathbf{e}\left[4 \operatorname{Re}\left\{\frac{1}{\sqrt{-3}} \operatorname{tr}\left((M+P) Q^{*}\right)\right\}\right] \\
& \times \mathbf{e}\left[\operatorname{tr}\left\{(M+P+A) \tau(M+P+A)^{*}+4 \operatorname{Re}\left((M+P) B^{*}\right)\right\}\right] \\
& =\sum_{P, Q \in L_{1} /\left(L_{1} \cap L_{2}\right)} \sum_{N \in L_{1}} \mathbf{e}\left[4 \operatorname{Re}\left\{\frac{1}{\sqrt{-3}} \operatorname{tr}\left(\left(V^{*} N+P\right) Q^{*}\right)\right\}\right] \\
& \quad \times \mathbf{e}\left[\operatorname{tr}\left\{\left(V^{*} N+P+A\right) \tau\left(V^{*} N+P+A\right)^{*}+4 \operatorname{Re}\left(\left(V^{*} N+P\right) B^{*}\right)\right\}\right] \\
& =\sum_{P, Q \in L_{1} /\left(L_{1} \cap L_{2}\right)} \sum_{N \in L_{1}} \mathbf{e}\left[4 \operatorname{Re}\left\{\operatorname{tr}\left(P B^{*}\right)\right\}\right] \mathbf{e}\left[4 \operatorname{Re}\left\{\frac{1}{\sqrt{-3}} \operatorname{tr}\left(N Q^{*} V^{*}\right)\right\}\right] \\
& \quad \times \mathbf{e}\left[\operatorname{tr}\left\{(N+V P+V A) \tau(N+V P+V A)^{*}+4 \operatorname{Re}\left(N B^{*} V^{*}\right)\right\}\right] \\
& \sum_{P, Q \in L_{1} /\left(L_{1} \cap L_{2}\right)} \mathbf{e}\left[4 \operatorname{Re}\left\{\operatorname{tr}\left(P B^{*}\right)\right\}\right] \\
& \times \sum_{N \in L_{1}} \mathbf{e}\left[\operatorname{tr}\left\{(N+V P+V A) \tau(N+V P+V A)^{*}+4 \operatorname{Re}\left(N\left(V B-\frac{V Q}{\sqrt{-3}}\right)^{*}\right)\right\}\right] .
\end{aligned}
$$

We put $e=(-1 / \sqrt{-3}) p, f=(1 / \sqrt{-3}) q$ for $p, q \in\{0, \pm 1\}^{r}$, then $\operatorname{tr}\left(P B^{*}\right)$ is expressed as $=-\sqrt{-3} e\left(b_{1}+b_{2}+b_{3}\right)^{*}$. Since

$$
V A=-\sqrt{-3}\left(\begin{array}{l}
a_{1} \\
a_{2} \\
a_{3}
\end{array}\right), \quad V B=-\sqrt{-3}\left(\begin{array}{l}
b_{1} \\
b_{2} \\
b_{3}
\end{array}\right), \quad V P=-\left(\begin{array}{l}
e \\
e \\
e
\end{array}\right), \quad V Q=\left(\begin{array}{l}
f \\
f \\
f
\end{array}\right)
$$

the last summation

$$
\sum_{N \in L_{1}} \mathbf{e}\left[\operatorname{tr}\left\{(N+V P+V A) \tau(N+V P+V A)^{*}+4 \operatorname{Re}\left(N\left(V B-\frac{V Q}{\sqrt{-3}}\right)^{*}\right)\right\}\right]
$$

becomes

$$
\prod_{j=1}^{3} \Theta\left(\begin{array}{c}
-e-\sqrt{-3} a_{j} \\
-\frac{f}{\sqrt{-3}}-\sqrt{-3} b_{j}
\end{array}\right)(\tau)=\prod_{j=1}^{3} \Theta\left(\begin{array}{c}
e+\sqrt{-3} a_{j} \\
\frac{f}{\sqrt{-3}}+\sqrt{-3} b_{j}
\end{array}\right)(\tau) .
$$

When $a_{1}=a, b_{1}=b, a_{2}=a_{3}=b_{2}=b_{3}=0$, the first equality of this theorem reduces to the second equality. 
Remark 3. The lattices $\sqrt{-3} L$ and $L_{1} \cap L_{2}$ are isomorphic to $\left(E_{6}\right)^{r}$ and $\left(E_{6}^{*}\right)^{r}$, respectively, where $E_{6}$ is the root lattice of type $E_{6}$, and $E_{6}^{*}$ is its dual.

To simplify cubic relations among $\Theta_{\omega}\left(\begin{array}{c}a \\ b\end{array}\right)(\tau)$, we put

$$
\begin{aligned}
\Theta_{\omega}\left[\begin{array}{l}
a \\
b
\end{array}\right](\tau) & =\Theta_{\omega}\left(\begin{array}{c}
\frac{a}{\sqrt{-3}} \\
\frac{b}{3}
\end{array}\right)(\tau) \\
& =\sum_{n \in \mathbb{Z}[\omega]^{r}} \mathbf{e}\left[2\left(n+\frac{a}{\sqrt{-3}}\right) \tau\left(n+\frac{a}{\sqrt{-3}}\right)^{*}+\frac{4}{3} \operatorname{Re}\left(n b^{*}\right)\right] .
\end{aligned}
$$

They satisfy:

(1) $\Theta_{\omega}\left[\begin{array}{c}\varepsilon a \\ \varepsilon b\end{array}\right](\tau)=\Theta_{\omega}\left[\begin{array}{l}a \\ b\end{array}\right](\tau)$ for a unit $\varepsilon \in \mathbb{Z}[\omega]$;

(2) $\Theta_{\omega}\left[\begin{array}{c}a+\sqrt{-3} p \\ b+\sqrt{-3} q\end{array}\right](\tau)=\mathbf{e}\left[-\frac{4}{3} \operatorname{Re}\left(p b^{*}\right)\right] \Theta_{\omega}\left[\begin{array}{c}a \\ b\end{array}\right](\tau)$ for $p, q \in \mathbb{Z}[\omega]^{r}$;

(3) $\Theta_{\omega}\left[\begin{array}{c}a \\ b\end{array}\right](T \cdot \tau)=\Theta_{\omega}\left[\begin{array}{c}-\bar{a} \\ \bar{b}\end{array}\right](\tau)=\Theta_{\omega}\left[\begin{array}{c}\bar{a} \\ -\bar{b}\end{array}\right](\tau)$.

COROLLARY 2. For $a, b \in \mathbb{Z}[\omega]^{r}$, we have

$$
3^{r} \Theta_{\omega}^{3}\left[\begin{array}{l}
a \\
b
\end{array}\right](\tau)=\sum_{p, q \in\{0, \pm 1\}^{r}} \mathbf{e}\left[-\frac{4}{3} \operatorname{Re}\left(p b^{*}+a q^{*}\right)\right] \Theta_{\omega}^{3}\left[\begin{array}{l}
p \\
q
\end{array}\right](\tau) .
$$

Proof. Theorem 3 implies that

$$
\begin{aligned}
& 3^{r} \Theta_{\omega}^{3}\left[\begin{array}{l}
a \\
b
\end{array}\right](\tau)=3^{r} \Theta\left(\begin{array}{c}
\frac{a}{\sqrt{-3}} \\
\frac{b}{3}
\end{array}\right)(\tau)^{3}
\end{aligned}
$$

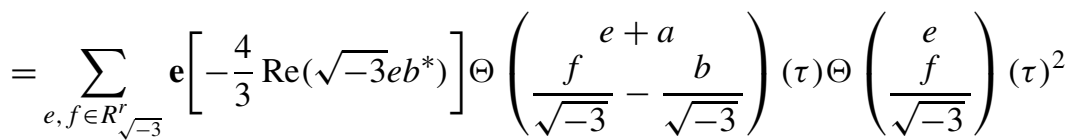

$$
\begin{aligned}
& =\sum_{e, f \in R_{\sqrt{-3}}^{r}} \mathbf{e}\left[-\frac{4}{3} \operatorname{Re}\left(\sqrt{-3} e b^{*}\right)\right] \Theta_{\omega}\left[\begin{array}{c}
\sqrt{-3}(e+a) \\
-\sqrt{-3}(f-b)
\end{array}\right](\tau) \Theta_{\omega}\left[\begin{array}{c}
\sqrt{-3} e \\
-\sqrt{-3} f
\end{array}\right](\tau)^{2} \\
& =\sum_{p, q \in\{0, \pm 1\}^{r}} \mathbf{e}\left[-\frac{4}{3} \operatorname{Re}\left(p b^{*}+a q^{*}\right)\right] \Theta_{\omega}^{3}\left[\begin{array}{l}
p \\
q
\end{array}\right](\tau),
\end{aligned}
$$

where we put $p=\sqrt{-3} e$ and $q=-\sqrt{-3} f$. 
Remark 4. In the equality of Corollary 2 , the coefficient of $\Theta_{\omega}^{3}\left[\begin{array}{l}-p \\ -q\end{array}\right](\tau)$ is the complex conjugate of that of $\Theta_{\omega}^{3}\left[\begin{array}{l}p \\ q\end{array}\right](\tau)$. If we eliminate one of $\Theta_{\omega}^{3}\left[\begin{array}{l}-p \\ -q\end{array}\right](\tau)$ and $\Theta_{\omega}^{3}\left[\begin{array}{l}p \\ q\end{array}\right](\tau)$ $((p, q) \neq(0, \ldots, 0))$ in this equality by the relation $\Theta_{\omega}\left[\begin{array}{l}-p \\ -q\end{array}\right](\tau)=\Theta_{\omega}\left[\begin{array}{c}p \\ q\end{array}\right](\tau)$, then the coefficient of $\Theta_{\omega}^{3}\left[\begin{array}{l}p \\ q\end{array}\right](\tau)$ for $(p, q)=(0, \ldots, 0)$ is 1 , and the others become -1 or 2 .

Proposition 2. If $a, b \in \mathbb{Z}[\omega]^{r}$ and $a b^{*} \notin \sqrt{-3} \mathbb{Z}[\omega]$ then $\Theta_{\omega}\left[\begin{array}{l}a \\ b\end{array}\right](\tau)=0$.

Proof. Note that

$$
1-\omega=\sqrt{-3} \omega^{2}, \quad \omega^{2}(1-(1-\omega))=1 .
$$

We have

$$
\begin{aligned}
\Theta_{\omega}\left[\begin{array}{l}
a \\
b
\end{array}\right](\tau) & =\Theta_{\omega}\left[\begin{array}{l}
\omega^{2}(1-(1-\omega)) a \\
\omega^{2}(1-(1-\omega)) b
\end{array}\right](\tau) \\
& =\Theta_{\omega}\left[\begin{array}{l}
a-\sqrt{-3} \omega^{2} a \\
b-\sqrt{-3} \omega^{2} b
\end{array}\right](\tau)=\mathbf{e}\left[\frac{4}{3} \operatorname{Re}\left(\omega^{2} a b^{*}\right)\right] \Theta_{\omega}\left[\begin{array}{l}
a \\
b
\end{array}\right](\tau) .
\end{aligned}
$$

Thus, $\Theta_{\omega}\left[\begin{array}{l}a \\ b\end{array}\right](\tau)=0$ if $\mathbf{e}\left[\frac{4}{3} \operatorname{Re}\left(\omega^{2} a b^{*}\right)\right] \neq 1$, which is equivalent to $a b^{*} \notin \sqrt{-3} \mathbb{Z}[\omega]$ for $a, b \in \mathbb{Z}[\omega]^{r}$.

We give examples of cubic relations among $\Theta_{\omega}\left[\begin{array}{l}a \\ b\end{array}\right](\tau)$ for $a, b \in \mathbb{Z}[\omega]^{r}$. For $r=1$, there are three non-zero $\Theta_{\omega}\left[\begin{array}{l}a \\ b\end{array}\right]$ on $\mathbb{D}^{1}=\mathbb{H}$ over $\mathbb{Z}[\omega]$ for $a, b \in\{0, \pm 1\}$ :

$$
\Theta_{\omega}\left[\begin{array}{l}
0 \\
0
\end{array}\right], \quad \Theta_{\omega}\left[\begin{array}{l}
1 \\
0
\end{array}\right]=\Theta_{\omega}\left[\begin{array}{c}
-1 \\
0
\end{array}\right], \quad \Theta_{\omega}\left[\begin{array}{l}
0 \\
1
\end{array}\right]=\Theta_{\omega}\left[\begin{array}{c}
0 \\
-1
\end{array}\right] .
$$

Corollary 2 implies the cubic relation among them:

$$
\Theta_{\omega}^{3}\left[\begin{array}{l}
0 \\
0
\end{array}\right](\tau)-\Theta_{\omega}^{3}\left[\begin{array}{l}
1 \\
0
\end{array}\right](\tau)-\Theta_{\omega}^{3}\left[\begin{array}{l}
0 \\
1
\end{array}\right](\tau)=0 .
$$

For $r=2$, there are 17 non-zero $\Theta_{\omega}\left[\begin{array}{l}a \\ b\end{array}\right]$ on $\mathbb{D}^{2}$ over $\mathbb{Z}[\omega]$ for $a, b \in\{0, \pm 1\}^{2}$. Corollary 2 implies linear relations among their cubes. The seven functions

$$
\begin{gathered}
\Theta_{\omega}^{3}\left[\begin{array}{c}
1,1 \\
1,-1
\end{array}\right], \Theta_{\omega}^{3}\left[\begin{array}{l}
1,1 \\
0,0
\end{array}\right], \Theta_{\omega}^{3}\left[\begin{array}{c}
1,1 \\
-1,1
\end{array}\right], \Theta_{\omega}^{3}\left[\begin{array}{l}
1,0 \\
0,1
\end{array}\right], \\
\Theta_{\omega}^{3}\left[\begin{array}{c}
1,-1 \\
1,1
\end{array}\right], \Theta_{\omega}^{3}\left[\begin{array}{l}
0,1 \\
1,0
\end{array}\right], \Theta_{\omega}^{3}\left[\begin{array}{l}
0,0 \\
0,0
\end{array}\right]
\end{gathered}
$$


are linearly independent in these relations. The rests can be expressed in terms of them:

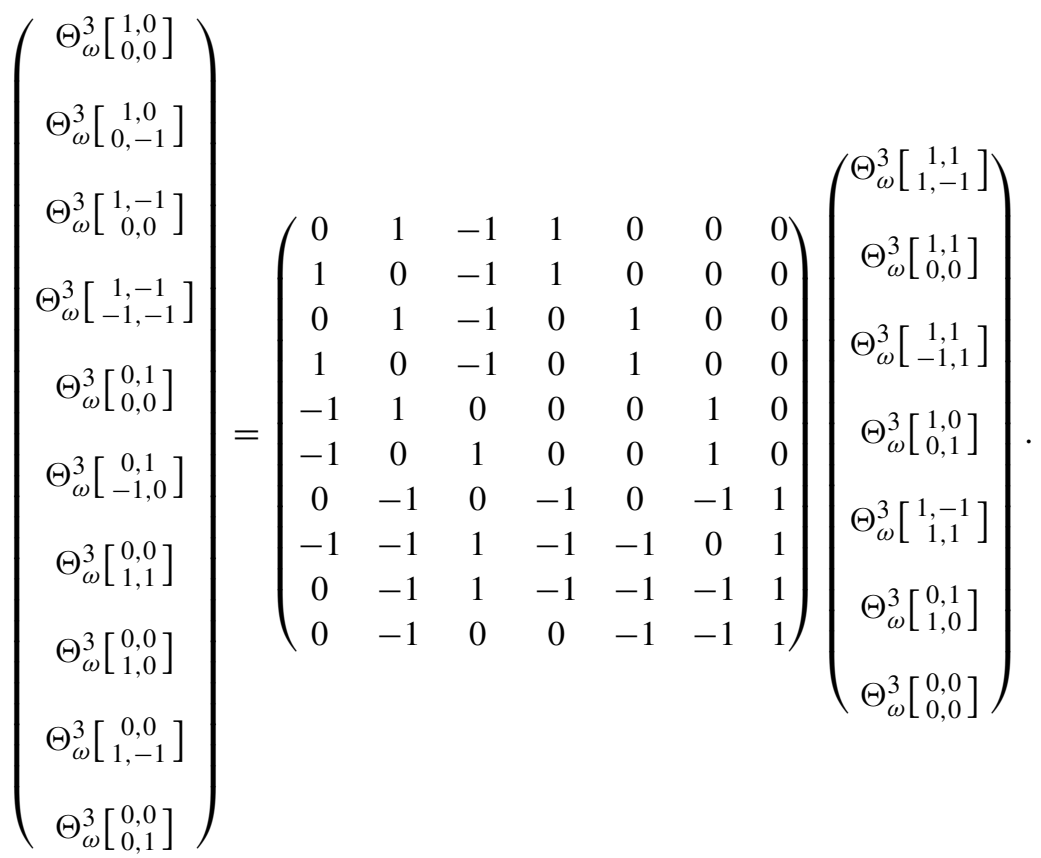

\section{REFERENCES}

[DK] T. Dern and A. Krieg. Graded rings of Hermitian modular forms of degree 2. Manuscripta Math. 110 (2003), 241-272.

[F] E. Freitag. Modulformen zweiten Grades zum rationalen und Gaußschen Zahlkörper. Sitzungsber. Heidelb. Akad. Wiss. 1 (1967), 1-49.

[FS] E. Freitag and R. Salvati Manni. Hermitian modular forms and the Burkhardt quartic. Manuskripte der Forschergruppe Arithmatik, Universität Mannheim, Heidelberg, 2003, pp. 1-24.

[I] J. Igusa. Theta Functions. Springer, Berlin, 1972.

[M] K. Matsumoto. Theta functions on the bounded symmetric domain of type $I_{2,2}$ and the period map of 4-parameter family of K3 surfaces. Math. Ann. 295 (1993), 383-408.

[MNY] K. Matsumoto, H. Nishi and M. Yoshida. Automorphic functions for the Whitehead-linkcomplement group. Preprint (2005).

[MY] K. Matsumoto and M. Yoshida. Invariants for some real hyperbolic groups. Internat. J. Math. 13 (2002), 415-443. 
Keiji Matsumoto

Department of Mathematics

Hokkaido University

Sapporo 060-0810

Japan

(E-mail: matsu@math.sci.hokudai.ac.jp) 